\title{
MODELO ECOSISTÉMICO DE EMPRENDIMIENTOS DINÁMICOS
}

\author{
Patricia Salcedo Camacho GE 1 Prado Lafuente Roger GE \\ Instituto de Investigación de la Carrera de Economía, Universidad Pública de El Alto, Villa Esperanza km \\ 15, Ciudad de El Alto, Bolivia \\ 1. Profesor de Administración del Talento Humano de la Carrera de Administración de Empresas, Uni- \\ versidad Pública de ElAlto, Bolivia, E-mail address:polotecnologico@gmail.com \\ 2. Profesor de Emprendimietnos en la Carrera Administración de Empresas, Universidad Pública de El \\ AltoBolivia,E-mail address:tecnopolo@gmail.com
}

\section{Resumen}

La presente investigación es el resultado del trabajo de investigación analítica, descriptiva de los diferentes agentes del ecosistema creado para la formación de empresas dinámicas, realizado a partir del año 2001, en la Ciudad de La Paz, donde a través de un método cuanti -cualitativo, se diseñó, un nuevo modelo ecosistémico que responda a las necesidades económicas del país y principalmente de los graduados de las universidades, porque son quienes están en condiciones de emprender por oportunidad y lograr una oferta de bienes y servicios, donde la transformación y agregación de valor a la producción primaria, implica el uso de la ciencia, la tecnología y la innovación como el instrumento fundamental del desarrollo productivo, este modelo eco sistémico establece los vínculos de asociación positiva, que produzcan sinergia y dinamicen la economía de manera virtuosa, con lo cual se concluyó que la aplicación del modelo debería ser monitoreada por una institución pública, que cuente con redes sólidas para lograr la cohesión de equipos de trabajo con alto compromiso y amplia creatividad.

Palabras Claves:

Ecosistemas, empresas dinámicas, potencial emprendedor, transformación y agregación de valor a la producción primaria.

\begin{abstract}
This research is the result of analytical research, descriptive of the different actors of the ecosystem created for the formation of dynamic, made from the year 2001 in the city of La Paz, where through a quantitative and qualitative method was designed, a new eco-systemic model that meets the economic needs of the country and mainly university graduates, because they are those who are able to undertake on opportunity and make an offer of goods and services, where the transformation and aggregation value to primary production, involves the use of science, technology and innovation as the fundamental instrument of productive development, this eco-systemic model establishes positive partnerships that produce synergy and revitalize the economy so virtuous, withwhich it was concluded that the application of the model should be monitored by a public institution that has strong networks to achieve the cohesion of teams with high commitment and broad creativity
\end{abstract}

Keywords:

Ecosystems, dynamic, entrepreneurial pote<ntial, processing and adding value to primary production. 


\section{1.- Introducción}

El actual modelo económico del país, según el anuario 2011 del Vice Ministerio de Ciencia y Tecnología (VMCyT), considera una política de:"oferta donde la transformación y agregación de valor a la producción primaria, implica el uso de la ciencia, la tecnología y la innovación como el instrumento fundamental del desarrollo productivo, sin embargo, hasta la fecha no se ha establecido un mecanismo de asociatividad y conformación de redes en un ecosistema de fomento y apoyo al emprendedor que consolide la implementación de estrategias de incorporación del conocimiento y aplicación de nuevas tecnologías de información y comunicación"[1] según lo dictamina la Constitución Política del Estado en la Sección IV, Artículo 103, donde se considera la creación de un Sistema Estatal de Ciencia y Tecnología que articule el trabajo sistémico del Estado, las universidades, las empresas productivas y de servicio, tanto públicas como privadas, las naciones y pueblos indígena originario campesinos, y el sistema financiero.

Un diagnóstico preliminar de las nuevas empresas dinámicas iniciadas por oportunidad, refleja la evolución del trabajo realizado por en el ecosistema emprendedor desde la ideación en nivel escolar, hasta la puesta en marcha con apoyo de instituciones bancarias. Sin embargo el impacto a nivel de desarrollo económico aún es reducido, porque las instituciones trabajan aisladas duplicando esfuerzos porque desconocen el trabajo de otros agentes del ecosistema. ${ }^{[2]}$

En función a estos antecedentes, se realizó una investigación profunda con objetivos trasformadores y emancipadores que tengan impacto en el corto, mediano y largo plazo, a través de tres grandes momentos: la sistematización de la información documental de las instituciones que trabajan en el fomento del emprendimiento dinámico, la entrevista a los responsables de cada organización para conocer sus necesidades, expectativas, vinculaciones de trabajo y percepciones; finalmente la reflexión del resultado de la asociatividad en el ecosistema emprendedor del país.

El presente artículo, presenta los resultados obtenidos de esta investigación cuanti-cualitativa, porque mide el impacto de las universidades y fundaciones que trabajan fomentando empresas dinámicas, así mismo describe percepciones, expectativas de al aplicar en la ciudad de La Paz, tomando en cuenta el modelo de ecosistema emprendedor del Babson Entrepreneurial
Ecosystem Project, propuesto por Daniel Isenberg.

\section{2.- OBJETIVOS}

\section{1.- OBJETIVO GENERAL}

Diseñar el mapa de vinculación de los agentes del ecosistema emprendedor de empresas dinámicas de la ciudad de La Paz.

\section{2.- OBJETIVOS ESPECÍFICOS}

Descripción de quienes son los potenciales agentes del ecosistema de empresas dinámicas que fomentan el emprendimiento en La Paz.

Evaluación de los agentes que trabajan en el ecosistema de empresas dinámicas, respecto a las actividades realizan y como se vinculan.

\section{3.- MATERIALES Y MÉTODO}

Para responder el nivel de profundidad al que se quiere llegar en la investigación y establecer las relaciones directas del ecosistema emprendedor, se utilizarán los siguientes métodos y técnicas científicas.

\section{Localización donde se realizó la investigación}

Departamento de La Paz, en oficinas de los agentes y asociados al ecosistema emprendedor: universidades públicas y privadas de la ciudad La Paz, financiadores, Ministerios y municipalidad, Fundaciones que trabajan en la Infraestructura del ecosistema y emprendedores que crearon su empresa entre el periodo comprendido del 2000 al 2010.

\section{Métodos para obtener el tamaño del universo de estudio}

Para alcanzar el objetivo se realizó entrevistas a 32 personas que dirigen los programas relacionados a empresas dinámicas, las cuales conforman el universo de investigación, que se clasifican en: instituciones que trabajan en la formación $\mathrm{y}$ fomento de un ecosistema de desarrollo productivo emprendedor, con actividades de sensibilización del espíritu emprendedor, concursos de modelo de negocios; Incubadoras, Aceleradoras de Empresas, redes, fondos de inversión, infraestructura de apoyo, que ofrecen el asesoramiento intelectual y recursos económicos para la puesta en marcha de los nuevos negocios.

En el caso de la sociedad civil se ha considerado como elemento común a todos los que fueron 
parte de procesos de capacitación, concursos e incubación en emprendimientos de base tecnológica, así mismo se realizó el seguimiento del impacto económico a través de entrevistas a empresas, instituciones públicas que confirmaron los datos estadísticos hallados por el $\mathrm{GEM}^{[3]}$ al 2010 .

\section{1.- Método Científico}

La investigación está elaborada en base al método deductivo, porque se partirá de las teorías generales de ecosistemas emprendedores y modelos de asociatividad para llegar al estudio y aplicación particular de los agentes del entorno.

El método propositivo, las técnicas de investigación para obtener información primaria y secundaria y la lista del universo de estudio se exponen en el anexo.

\section{Procedimientos empleados}

La información primaria se obtendrá de los agentes involucrados en el desarrollo emprendedor en la ciudad de La Paz, mediante entrevistas estructuradas a directivos de las instituciones que sean agentes potenciales de fomento a las empresas dinámicas.

La información secundaria se referirá a las memorias y otras publicaciones de industrias o instituciones que aportan datos estadísticos e históricos sobre el tema, la característica fundamental de la investigación en estos dos pasos, es el descubrimiento de principios generales, que coadyuvan al desarrollo de la investigación.

\section{2.- Técnicas de Investigación}

Se aplicó la técnica de observación participante, en donde al investigar se compartió con los investigados (objetos de estudio según el cánon positivista) para entender el contexto del ecosistema emprendedor, la experiencia y trabajo realizado, para obtener directamente toda la información que poseen los sujetos de estudio sobre su propia realidad, o sea, pretender conocer la el trabajo desde el interior del mismo.

Una vez identificado los potenciales agentes que influyen en el ecosistema emprendedor se aplicó la técnica de entrevista con un formulario que permita identificar actividades, necesidades de vinculación, expectativas en La Paz y los resultados obtenidos.

Esta información se consolidó en tablas de información cualitativa y gráficos que resumen el impacto y los años de experiencia.

\section{4.- MODELO ECOSISTÉMICO}

"Hacia el año 2010 se ha comprobado que países como Ruanda, Chile, Israel, e Islandia son un terreno fértil para la iniciativa empresarial, gracias en gran parte a los esfuerzos de sus gobiernos."[4] Donde los resultados y el impacto en los negocios son muy rentables.

Los expertos en temas empresariales afirman que estos ecosistemas de emprendedores se han convertido en una especie de alternativa para los gobiernos de todo el mundo, tanto en los países emergentes y en desarrollo. Por desgracia, muchos gobiernos adoptan un enfoque equivocado para construir ecosistemas empresariales. Persiguen un ideal inalcanzable de un ecosistema y ven a las economías que son completamente diferentes de las suyas las mejores prácticas.

Pero cada vez más, las prácticas más eficaces provienen de los rincones más remotos de la tierra, donde los recursos, donde "los casos de éxito destacan que el trabajo del gobierno involucra al sector privado y sin fines de lucro también para que también asuman parte de la responsabilidad."[5]

En el último ranking de facilidad para hacer negocios del Banco Mundial, se destaca esta evidencia de cambio del ecosistema de fomento al emprendedor, como menciona Isenberg: “...de un país diezmado por el genocidio en la década de 1990. En la lista del Banco Mundial, Ruanda catapultó fuera de la zona de Haití, Liberia y la Ribera Occidental y Gaza, y pasó junto a Italia, la República Checa, Turquía y Polonia. Por un subíndice en el estudio, la facilidad de abrir un nuevo negocio, Ruanda el puesto 11 a nivel mundial. Este es el tipo de cambio empresarial puede aportar a un país. Como presidente de Ruanda, Paul Kagame, dijo recientemente, "El espíritu empresarial es la forma más segura de desarrollo.” (ISENBERG, Daniel, 2012). Así mismo, el cambio económico por el desarrollo de nuevos negocios debe considerar las mejores prácticas desarrolladas en el tema, como lo sugiere Isenberg: "Para encender la creación y el crecimiento en participación, los gobiernos deben crear un ecosistema que sustenta los empresarios. Esto es lo que realmente funciona."

Considerando estos antecedentes la investigación se inició el año 2000 en La Paz, con el fin de profundizar en el estudio de las variables 
asociadas al proceso de creación y dinámica de las nuevas empresas creadas en el país. Estableciendo que el problema principal del ecosistema nacional es que en diez años aún no vincula los esfuerzos de manera sistemática, como lo mencionan estudios económicos de todo el mundo donde se halló una vinculación sistemática entre diferentes instituciones que trabajan fomentando el espíritu empresarial, demostrando rápido crecimiento de empleo, así como el crecimiento del PIB, y/o el aumento de la productividad a largo plazo.

\section{5.- RESULTADOS Y DISCUSIÓN}

El trabajo realizado en la Red Nacional de Incubadoras (RNI),dependiente del VMCyT en la gestión 2011 y 2012 tiene como objetivo general:

Proponer un Mapeo de los agentes del ecosistema emprendedor de la ciudad de La Paz en términos de asociatividad y conformación de redes para el fomento y apoyo de empresas dinámicas.

El mapa permitirá resolver el problema de coordinación que enfrenta la RNI, siendo una de las causas: el insuficiente tratamiento de la información de sus miembros y otros actores externos que trabajan, que impide contar con un panorama claro y preciso que informe en qué etapa del proceso emprendedor trabaja cada institución, qué experiencia tiene, cuales son los factores críticos de articulación con otras redes y qué entrega cada participante para alinear esfuerzos a favor de un ecosistema emprendedor

Para alcanzar el objetivo se realizó entrevistas a 32 personas que dirigen los programas relacionados a empresas dinámicas, las cuales conforman el universo de investigación propuesto por el VMCyT, que se clasifican en: instituciones que trabajan en la formación y fomento de un ecosistema de desarrollo productivo emprendedor, con actividades de sensibilización del espíritu emprendedor, concursos de modelo de negocios; Incubadoras, Aceleradoras de Empresas, redes, fondos de inversión, infraestructura de apoyo, que ofrecen el asesoramiento intelectual y recursos económicos para la puesta en marcha de los nuevos negocios.

El método propositivo, las técnicas de investigación para obtener información primaria y secundaria y la lista del universo de estudio se exponen en el anexo 13. Así mismo el trabajo cumplió los siguientes objetivos específicos:

- Descripción de quienes son los potenciales agentes que fomentan el emprendimiento, y pueden ser parte del ecosistema de empresas dinámicas.

- Evaluación de los agentes que trabajan en el ecosistema de empresas dinámicas, respecto a las actividades realizan y como se vinculan.

- Análisis de los modelos de integración utilizados, según la percepción de los entrevistados.

\section{1.- DESCRIPCIÓN DE LOS AGENTES DEL ECOSISTEMA}

En la primera fase de gestación: Los agentes del ecosistema emprendedor en La Paz, a la fecha son: diferentes Universidades desde la UCB - MPD, con mayor tiempo realizando cursos y actividades de sensibilización en temas de emprendimiento, junto con la UNSLP que llegó a asesorar algunas empresas dinámicas que surgieron por su materia transversal (más detalles ver anexo ), siendo las más antiguas en el ecosistema, con menor experiencia participan otras universidades desde insertar la materia de emprendimientos en la curricula, crear la modalidad de graduación por planes de negocio, hasta apoyar a estudiantes para concursos de ideas emprendedoras.

En la fase de puesta en marcha o lanzamiento de la nueva empresa se encuentran las Instituciones de apoyo como son: Fundación Nuevo Norte, con mayor experiencia trabajando con emprendedores artesanales y productores, así como en apoyo de gestión a ganadores del concurso Ideas Emprendedoras, Fundación MAYA que apoya a jóvenes universitarios que participa del concurso INNOVA SAN ANDRES, el área de Responsabilidad Social de SOBOCE con el concurso Emprende Ideas y los Centro de Innovación Tecnológica CITE para emprendedores en confecciones y madera, donde brinda servicios de capacitación, asistencia técnica - productiva y de gestión, mejorando la productividad y competitividad de las MIPYMES en las distintas etapas de la cadena productiva.

Los más recientes en el ecosistema son: Incubadora Ifaro e Incubadora HUB 7, que gestionan la puesta en marcha de ideas en software e informática, (más detalles ver anexo), también apoyan en capacitación y evaluación en el concurso STARTUP.BO, el cual se realiza por dos días en el cual estudiantes universitarios se reúnen por equipos para diseñar un modelo de negocio que exponen a un jurado evaluador.

En la fase de desarrollo inicial se encuentran: La 
Incubadora GAMLP, que asesora a emprendedores que comenzaron a vender sus servicios productos y el programa de Empresas Dinámicas. Así mismo, se encuentra el FONDO PARA LA INNOVACIÓN que provee de tecnologías financieras para los emprendedores.

Finalmente los agentes que coordinan y trabajan de manera integral son las redes RBE, a nivel privado, mientras que a nivel gubernamental está la RBI el Vice ministerio de Ciencia y Tecnología.

La siguiente figura resume a los potenciales agentes del ecosistema de fomento a empresas dinámicas, desde la academia, la infraestructura de apoyo y las intuiciones gubernamentales, rodeados de las empresas creadas.

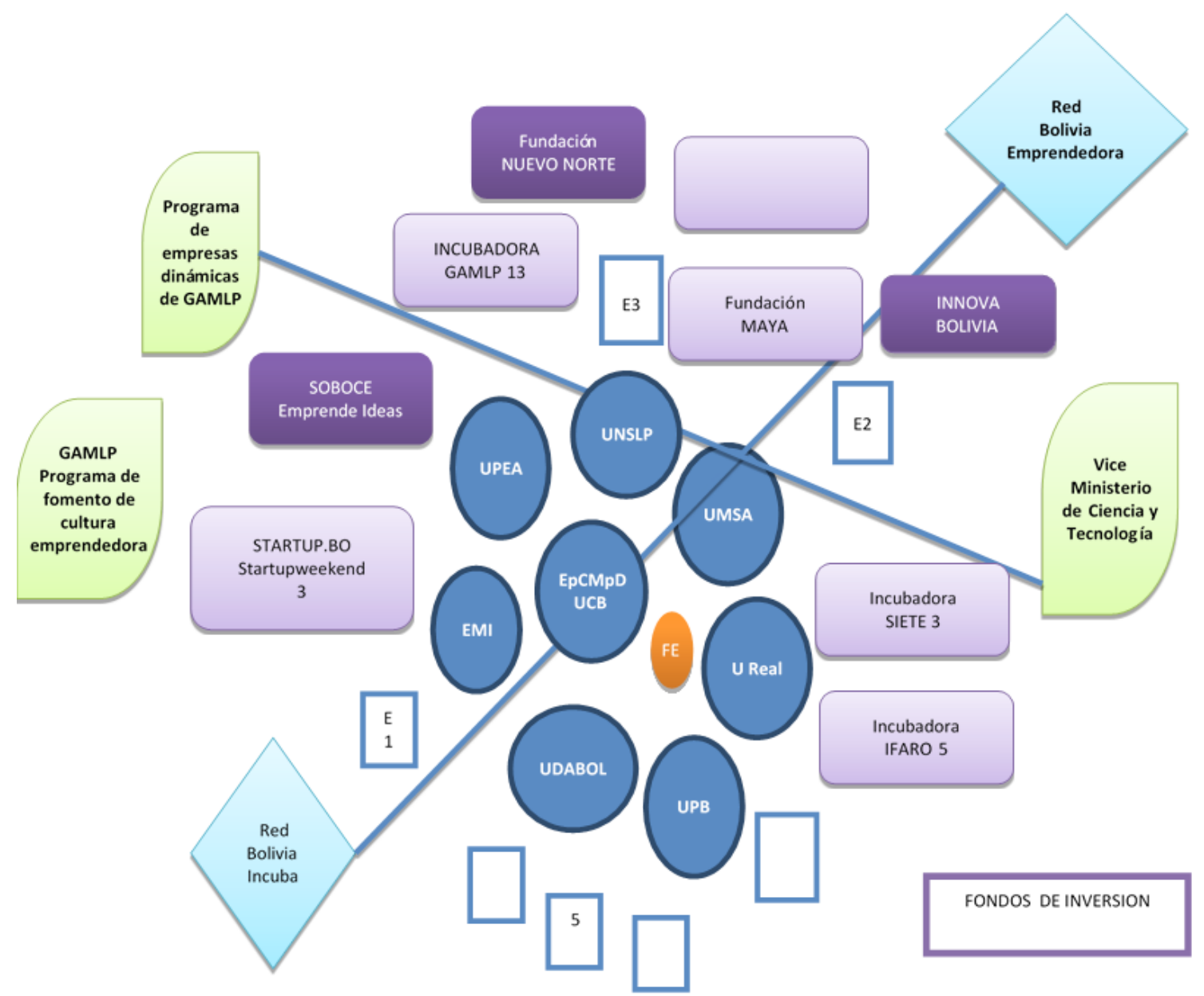

Fuente: Elaboración Propia en base a los agentes documentados en la investigación 2012

Gráfico 1.- Agentes del ecosistema la paz

Evaluación del Trabajo de los agentes del Ecosistema

Una vez identificado el panorama del ecosistema emprendedor se realizó la entrevista a los agentes propuestos por el VMCyT, para que evalúen su trabajo, y respondan a diferentes preguntas que buscan interpretar la percepción del trabajo que realizaban los demás agentes, tanto de universidades, como de instituciones de apoyo e infraestructura del ecosistema emprendedor, los resultados obtenidos se presentan resumidos en el siguiente gráfico: 


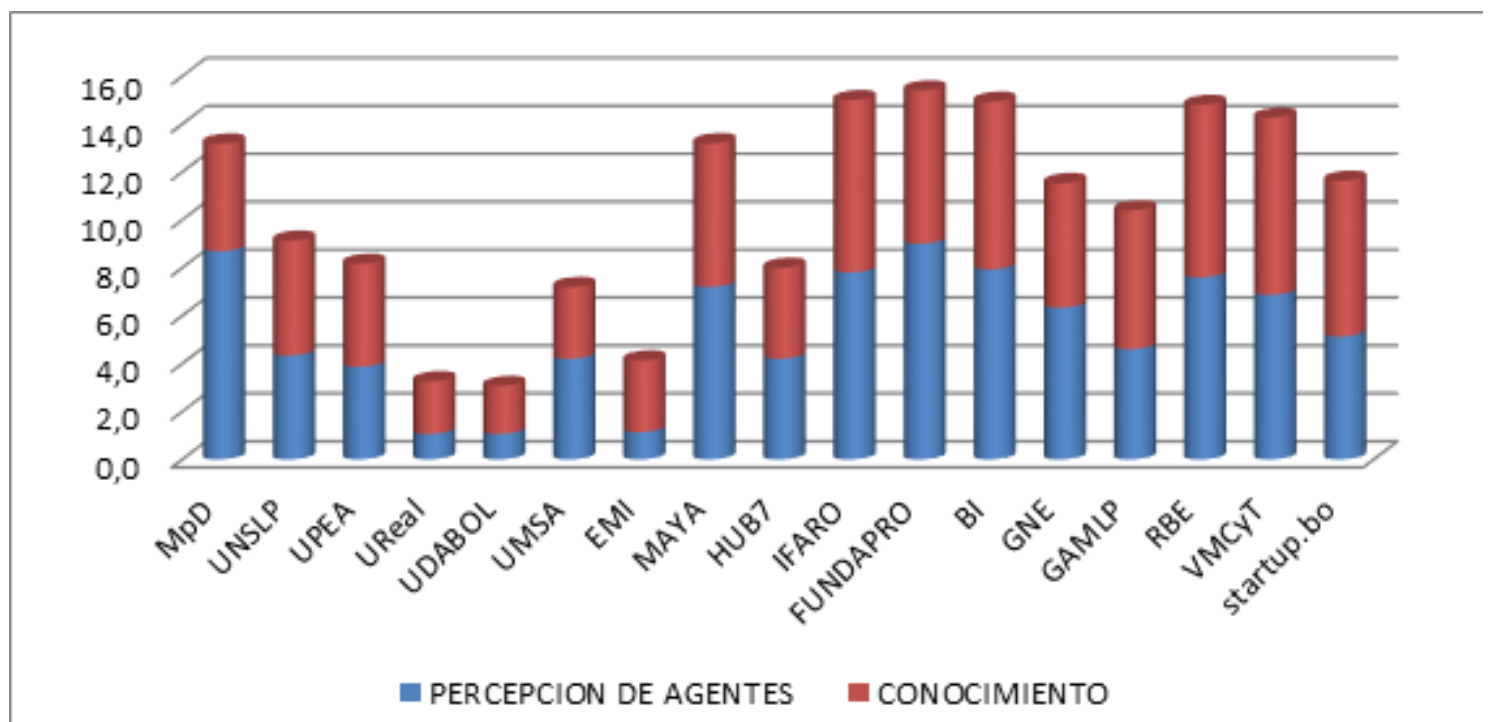

Fuente: Elaboración Propia en base a entrevistas a los agentes al 2012

Gráfico 2.- Evaluación de las Instituciones del Ecosistema Emprendedor de la Paz.

En las entrevistas se ha considerado una escala del 1 al 10 para las siguientes variables de evaluación considerando:

- Cómo es percibida cada institución en términos de importancia y conocimiento acumulado, acerca de Empresas Dinámicas en color azul

- El nivel de interrelacionamiento y la facilidad de trabajar con las instituciones mencionadas, en color rojo.

En la evaluación de percepción se observa que $\mathrm{MpD}$ es la institución académica mejor percibida por el ecosistema, en cuanto a los procesos de pre incubación e incubación.

Las FundacionesIfaro y Maya gozan de la confianza de los otros agentes, tanto en la academia como en los emprendedores que mencionan sus esfuerzos.

El concursoSTARTUP.BO y la Incubadora de la GAMLP, son nuevos en el ecosistema tienen un buen nivel de interrelacionamiento para prmocinar sus resultados y elevar la percepción del ecosistema respecto al apoyo de empresas dinámicas como ícono de la ciudad.

El trabajo realizado por la FUNDAPRO por sus años de experiencia y amplia gama de programas es la institución mejor percibida en Infraestructura de apoyo; así mismo laRBE, RBI y el VMCyT, son percibidos como muy importantes tanto en concimiento acumulado, así mismo el interrelacionamiento y la facilidad de trabajar con ellos, permite concluir que el encadenamiento promueve la alineación de esfuerzos, en el ecosistema, por lo cual están empezando a dar frutos en mayor número de empresas dinámicas.

En el conocimiento se observó la existencia de diversas instituciones trabajando en el tema emprendedor, sin un ente centralizador, que promueva un nuevo ecosistema emprendedor, donde las personas y el talento sean impulsados a través de reuniones para socializar sus problemas y sus expectativas.

Descripción de impactos de los agentes del ecosistema emprendedor

En cuanto a la formación emprendedora, se ha trabajado en términos de sensibilizar a la población en términos de fomentar pensamiento y espíritu emprendedor, a través de diferentes componentes y con diferentes énfasis, lo cual se destaca en el impacto de la sociedad: "quien ha dejado de ver al emprendedor como una persona informal; actualmente se considera al joven emprendedor como una persona con potencial para la generación de empleo" según los comentarios de los representantes de los concursos tanto del Gobierno Municipal de La Paz, como ferias en las universidades y colegios.

\section{Impacto en la academia}

Las universidades están transversalizando el espíritu emprendedor, desde diferentes niveles $\mathrm{y}$ con distintos fines, desde modalidades de 
graduación en carreras de negocios, mientras que en carreras como Ingeniería e Informática es una forma de patentar sus innovaciones y lograr ingresos por sus prototipos o investigaciones, según los agentes de la academia entrevistados, sin embargo, los resultados obtenidos hasta la fecha son mínimos comparado al número de graduados, con un indicador de 1 de 20 que llevó al mercado su plan de negocio, en promedio el $5 \%$ son empresas dinámicas.

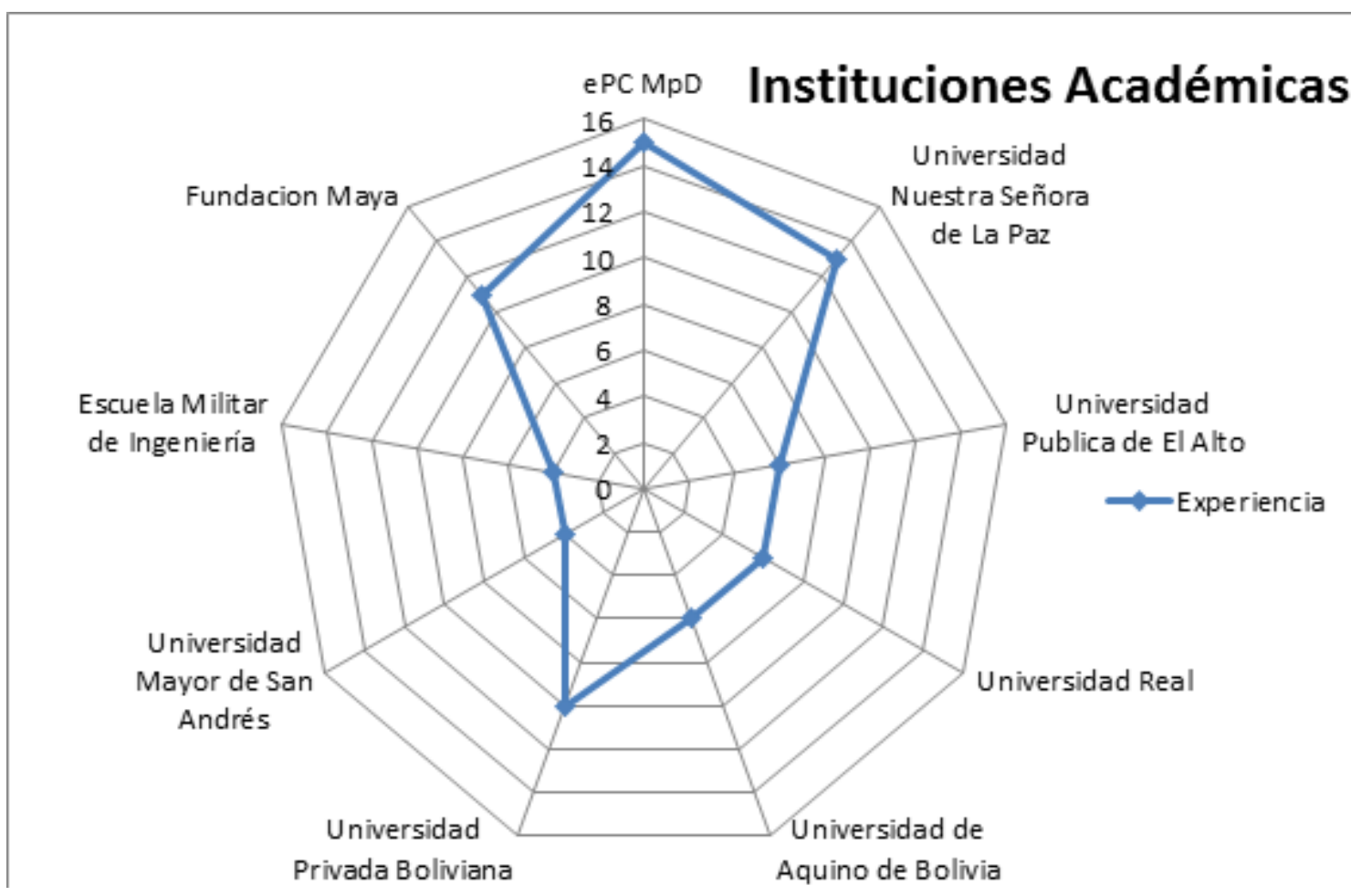

Fuente Elaboración propia en función a datos obtenidos en la entrevista.

Gráfico 3.- Años de experiencia de la academia.

$\mathrm{MpD}$, es la que mas experiencia tiene en la temática porsus 15 años de experiencia en la implementación de su catedra de emprendimientos, cursos especiales con especialistas de la región en plan de negocios y empresas dinámicas, investigación relacionada al emprendimiento como el Global Entrepreneurship Monitor Bolivia, y libros escritos acerca de la temática en el país, además de participar de foros de importancia mundial; UNSLP, con 13 años de experiencia en el área de la pre incubación y convenios con Emprendia y asesoría de incubadoras como la del director de la incubadora social del Tecnológico de Monterrey; UPB, modelo su sistema de incubación como parte de trabajos de tesis de maestría y se especializo en dar capacitación en emprendimientos en sectores vulnerables, municipios y otros, la fundación Maya a través del Centro de INvestigacion y Desarrollo Emprendedor de INgenieria Industrial de la UMSA, tamnbien esta trabajando hace 10 años en la asesoría de planes de negocio y la sensibilización de emprendiientos en las carreras de INgenieria, el resto de universidades aun son noveles en la enseñanza de emprendimientos pero están generando conocimiento y experiencia.

Como se observa en la figura 6, las nuevas instituciones con menos de cinco años de experiencia, han logrado impactos representativos en segmentos específicos con los que pretenden demostrar resultados de empresas, productos y apertura de mercados, que por el momento son escépticos a la calidad y experiencia de los emprendedores, los entrevistados afirman que lo más importante es cambiar el comportamiento del consumidor y crear una cultura de falla para probar conceptos de mercado antes de establecer empresas no acordes a los cambios dinámicos, por esta razón han cambiado su forma de estructurar la ideas de negocio en modelación y prototipación para pasar en una segunda instancia al plan de negocio.

La industria de soporte es crucial para el desarrollo de un ecosistema emprendedor, FUNDAPRO, ha generado conocimiento $\mathrm{y}$ experiencia en las áreas de financiamiento e incubación a través del programa de fomento a la cultura 
emprendedora, desde el Fondo Emprender, la incubadora NEOEMPRESA, y ahora el fondo para la Innovación, con 12 años de experiencia es un referente importante acerca del trabajo que se hizo, que cosas funcionaron, y que se necesitan implementar; la Red Boliva Emprendedora, trabaja en apoyar a los programas emprendedores desde su conocimiento de los agentes y los primeros esfuerzos de interrelacionamiento; el concurso de Ideas Emprendedoras, con una media los últimos años de 3000 ideas de negocio presentadas es también un buen referente con sus
7 versiones del concurso para conocer la intención del Boliviano para emprender; en los últimos 5 años han comenzado a operar otras iniciativas importantes en el campo de la incubación como IFaro, HUB7 y la incubadora de la GAMLP, y la creación de redes más específicas de apoyo a emprendedores como la Red Bolivia Incuba, VMCyT involucrando al gobierno estatal en la temática emprendedora; startup.bo a través del startup weekend y el concurso emprende ideas dan mas opciones a los emprendedores para probar sus ideas y recibir retralimentacion para mejora.

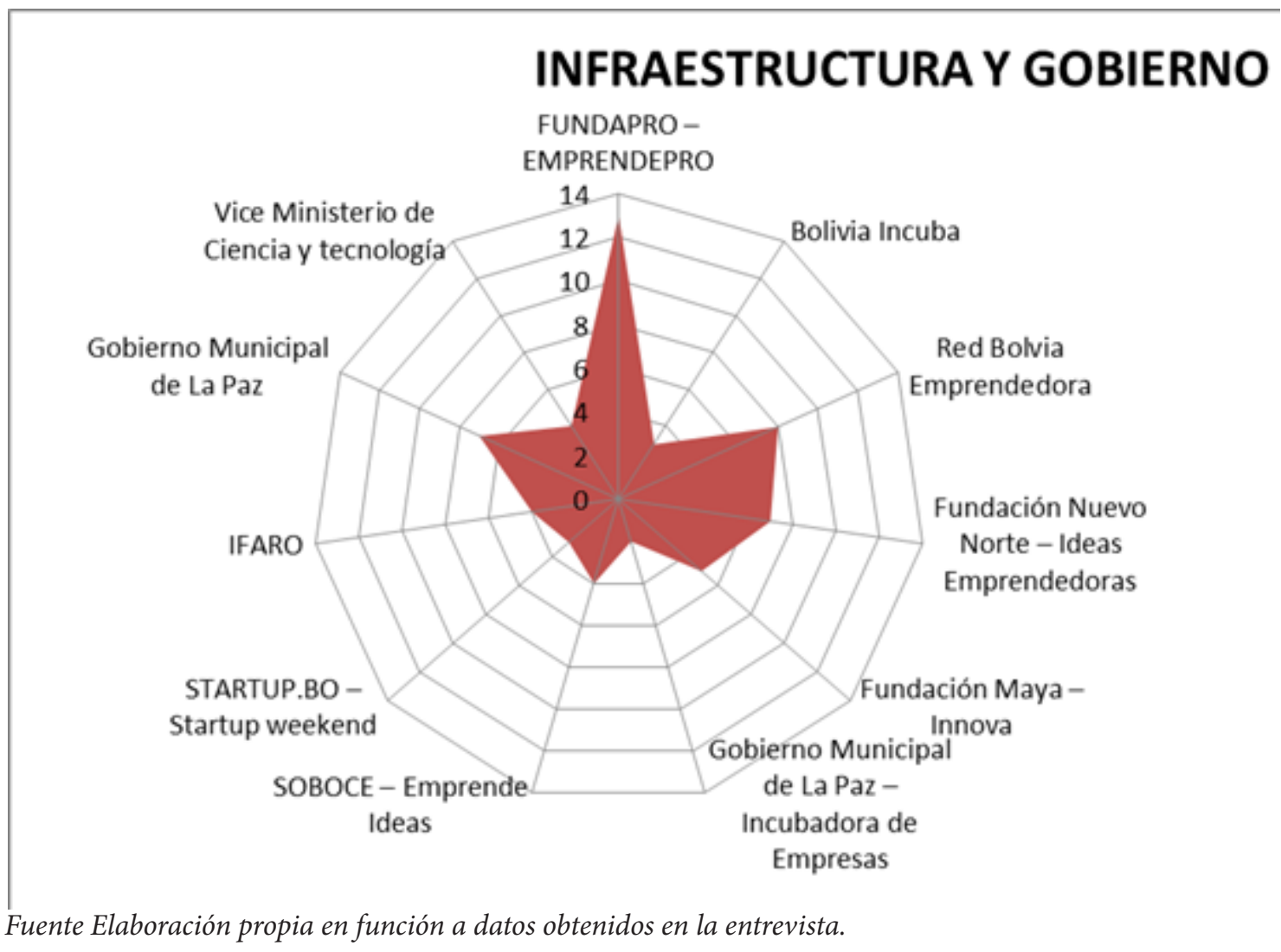

Gráfico 4.- Años de experiencia de Instituciones

Según las entrevistas realizadas, las instituciones académicas han comenzado a capacitar y utilizar el modelo CANVAS ${ }^{[6]}$, como formato estándar para la presentación de ideas de negocio, en diferentes concursos y programas, esto ha logrado homogenizar la transversalización de conocimientos de negocios dinámicos, desde la EpC UCB, UMSA, UNSLP hasta modelos de trasferencia que realizan la alcaldía de La Paz y la Red Bolivia Incuba. Así mismo la graduación por planes de negocio, en la UMSA y la UCB está motivando a que los nuevos profesionales generen capacidades de aterrizar las ideas de negocio en documentos para iniciar procesos de formalización de empresas dinámicas.
El impacto de la academia en La Paz, en estos diez, años se mide en el capital intelectual del ecosistema, el cual actualmente está sostenido por más de cien profesionales con conocimiento en: modelos de trasferencia para aceleración; modelos de impulso al proceso emprendedor y modelos de incubación de empresas, como el Modelo de Incubación Robusta (MIR) del Instituto Politécnico Nacional de México; así mismo, varios docentes han visitado congresos Internacionales de emprendedores organizados por BabsonCollege, HARDVARD y Tecnológico de Monterrey de México, para actualizarse en el estado de la cuestión y en planes de negocio como CEFE, ISUM de la IOT. El resultado de 
éxito es más de cinco mil personas capacitadas y entrenadas en estos temas.

Respecto a la vinculación entre instituciones que trabajan en el tema, se ha identificado un bajo nivel de asociación y sinergia entre ePCMpD, UMSA,FUNDACION MAYA, Universidad REAL, EMI, UNSLP, UDABOL, UTB, UE, AGENCIAS DE COOPERACION, FUNDAPRO, Fundación IFaro, RED BOLIVIA INCUBA, quienes si bien conocen la importancia de trabajar de manera conjunta en actividades organizadas por la Red Bolivia Emprendedora, el evento de startup.bo, el concurso Innova Bolivia, aún no se ha consolidado el trabajo por objetivos comunes, por la ausencia de reuniones mensuales en un espacio definido que propicien un intercambio de experiencias y problemas, que fortalezca la convivencia social.

Sin embargo cabe destacar la sólida relación entre EpC UCB con GAMLP que están creando una marca ciudad emprendedora, con infraestructura y programas de fomento al ecosistema emprendedor; así mismo otra vinculación exitosa es el VCMCyT con IFaro, quienes han logrado incidir en la Ley de Telecomunicaciones, con el fin de abrir mercado para los programadores de aplicaciones en software libre.

Según Cecilia Valda del Programa de Pre incubación de la UNSLP, la necesidad de las universidades privadasen ocasiones se resume a: "Lo que hace falta es ideas y plata"; "la universidad está en el centro no es la que más necesita, ni la que tiene más, pero los fondos siempre buscan a los más pobres".

Mientras que para Carla Gutiérrez lo que realmente necesita el ecosistema es que se institucionalice el trabajo y la vinculación, que deje de depender las relaciones de la amistad y el contacto social. "porque luego uno cambia de trabajo y las cosas se frenan”.

Asimismo, según Gonzalo Galleguillos, lo que el ecosistema necesita es cooperar y que dejen de centralizar en una institución todas las etapas: "es necesario que deje de existir personas y que quieran hacer solitos todo el trabajo, y que aprendan a trabajar con roles, para ser más efectivos".

Finalmente, Diego Velarde afirma la principal necesidad del ecosistema radica en: "la alineación y objetivos y concentrar esfuerzos de cada agente".
Por tanto, la conclusión común de los agentes, respecto al éxito del ecosistema emprendedor en La Paz, radica en cerrar la brecha común que impide que se desarrollen mas empresas dinámicas, un común denominador que es el capital operativo en la etapa inicial, es decir, la escasez de fondos de inversión para empresas en etapa cero, es decir idea de negocio, inicial ver lo anteriormente expuesto en Kantis.

- Las instituciones públicas remarcaron la necesidad de hacer más sencillos los procesos de incubación y de recepción de ideas de negocio, las cuales actualmente tienen procesos no amigables para los interesados, y el exceso de burocracia desmotiva emprendedores a continuar trabajando dentro de la institución.

Finalmente, se puede destacar que se está modificando el enfoque del cliente objetivo del fondo de inversión, de FUNDA PRO, quienes después de haber analizado la experiencia del Fondo Emprender -que estaba destinado al escalamiento de negocios, con préstamos mayores a 50.000 dólares americanos, en un mercado emprendedor que requería cantidades menores- el nuevo enfoque permitirá el acceso de empresas con seis meses de trabajo, para obtener financiamiento de capital de operación, más accesible y ajustado a su actividad, desde los 6 meses de actividad y ya con operaciones comerciales.

Este fondo está iniciando operaciones, y es coordinado por personal con conocimiento de los agentes y sus necesidades, es una apuesta a lo que el resto del ecosistema ha estado atribuyendo como el problema principal, que era la falta de fondos en la gestación de la idea.

\section{6.- ANÁLISIS DE LOS MODELOS DE ECOSISTEMAS}

Según documentos obtenidos se identifica dos modelos de ecosistema emprendedor desarrollados en la ciudad de La Paz en la última década, a continuación se analizarán los componentes de cada uno, los agentes que participaron y sus experiencias.

\subsection{Modelo Cadena de Valor}

El programa Fondo Emprender, fue el pionero en proponer un ecosistema que integre el esfuerzo y trabajo de diferentes instituciones, el cual estaba organizado en cuatro componentes: 
- Estímulo a la formación de ideas y planes de negocio.

- Apoyo a la implementación y desarrollo de nuevas empresas

- Financiamiento de nuevas empresas

- Aprendizaje y difusión de resultados y propuestas de política.

Este modelo se estructuró bajo la concepción de una cadena de valor, es decir, cada institución realizaba un trabajo gradual, para dar insumos al siguiente agente, de esta manera los emprendedores pasaban las tres etapas de Kantis, desde la sensibilización, hasta el financiamiento de nuevas empresas, buscando una sinergia a través de todo el proceso, con instituciones especializadas en cada etapa, como se presenta en la siguiente figura:

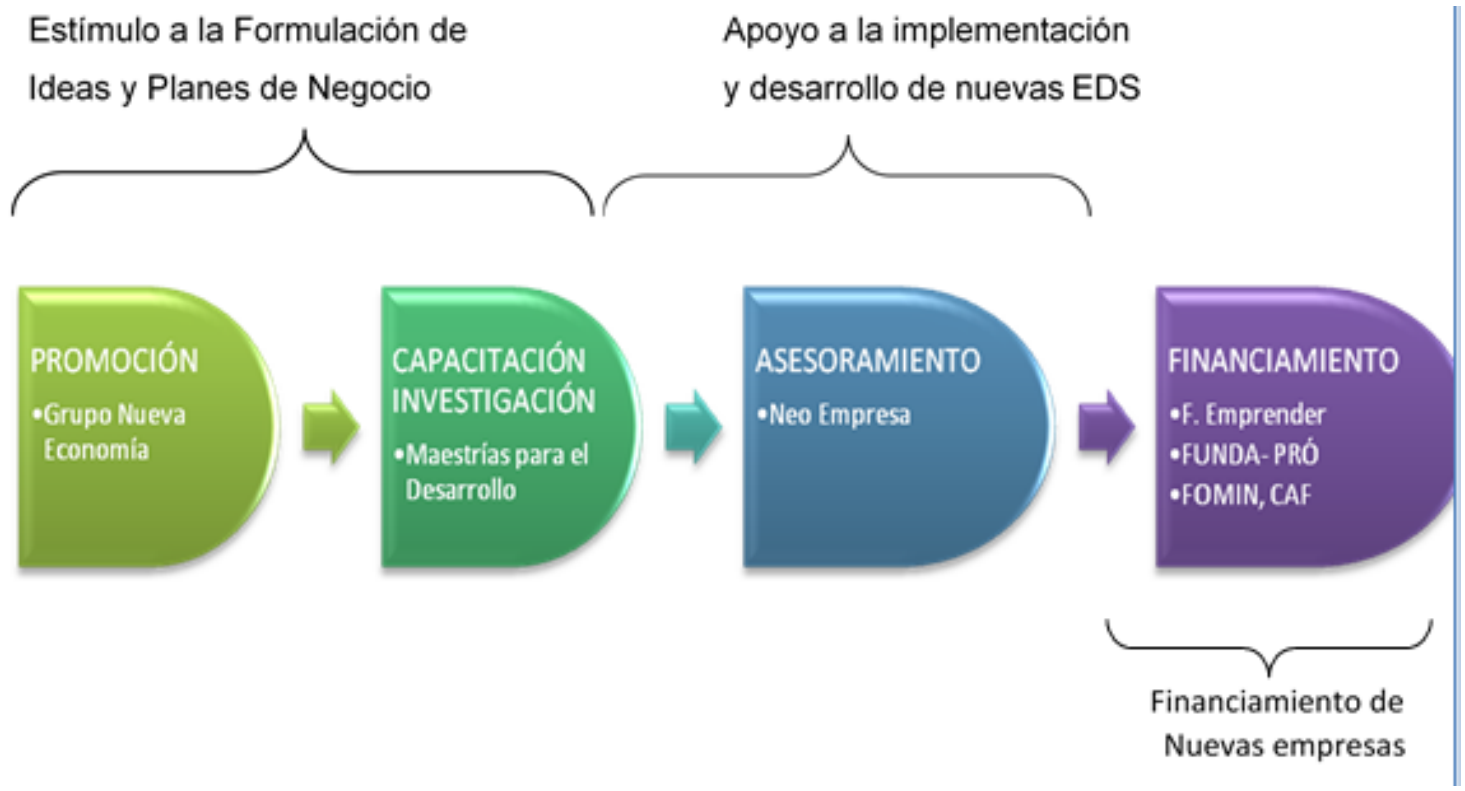

Gráfico 5.- Cadena de Valor Programa Fomento de Cultura Emprendedora

Sin embargo, los cambios políticos - con la izquierda en el poder al mando de Evo Moralesy las relaciones gubernamentales con organismos internacionales ${ }^{[7]}$ afectaron este programa, el cual hasta el 2009 trabajo con la incubadora NEOEMPRESA realizando las primeras alianzas con otros agentes del ecosistema emprendedor con la intención de sostener en el largo plazo ciertas empresas modeladas por oportunidad; no obstante, según la entrevista realizada a Gonzalo Gallegillos[8]: “los altos estándares y restricciones - establecidas por los organismos institucionales que daban el financiamientolimitaban el número de empresas incubadas, no por falta de recursos, ideas o clientes, sino por la coyuntura político económica"

Así mismo, según documentos obtenidos de la memoria del programa de Fomento a la Cultura Emprendedora al 2010, se destaca la revisión de resultados parciales, donde se cita textualmente: "a mediados del 2009 se identificó - dentro de la cadena de valor- no se había establecido un vínculo adecuado entre cada una de las organizaciones, el proceso no apuntó a un acercamiento más profundo entre las instituciones o personas; pero permitió identificar mecanismos concretos de articulación de las actividades." Con lo cual se puede afirmar la importancia de la atracción y retención de talento interno de cada institución, para que el ecosistema genere impactos de largo plazo, donde todos los agentes están alineados a una visión común, que integra el trabajo de cada institución como un todo armónico en lugar de trabajar en todas las etapas de manera aislada.

\section{2.- Modelo de nodos de Instituciones Privadas}

Después del trabajo del programa del Fondo Emprender, el país ingresa a un período de formación masiva en espíritu emprendedor, en diferentes segmentos de la población, sensibilizadas con el espíritu emprendedor y la generación de ideas de negocio, esto es medido el 2008 en informes de Global Entreprenuership Monitor, donde Bolivia participa por primera vez y manifiesta que existe un alto porcentaje de la población iniciando un emprendimiento, aunque en la mayoría de los casos se trata de emprendimientos motivados por la necesidad, 
que no están generando nuevos empleos y el negocio participa en mercados tradicionales de comercialización de alimentos y mercancía importada o servicios técnicos, como lo menciona Fernández:

"Una gran proporción de estas iniciativas operan en la informalidad y son abandonadas si la persona que los lidera obtiene un trabajo asalariado de tiempo completo."

No obstante, al existir mayor sensibilización del tema, los emprendimientos por oportunidad comienzan a ser parte del interés de otras instituciones, tanto académicas, gubernamentales y sociedad civil que se integran en nodos locales de la Red Bolivia Emprendedora aglutinando esfuerzos y vinculando procesos para fortalecer empresas dinámicas, desde un centralización en la ciudad de La Paz, desde el 2005 como se gráfico:

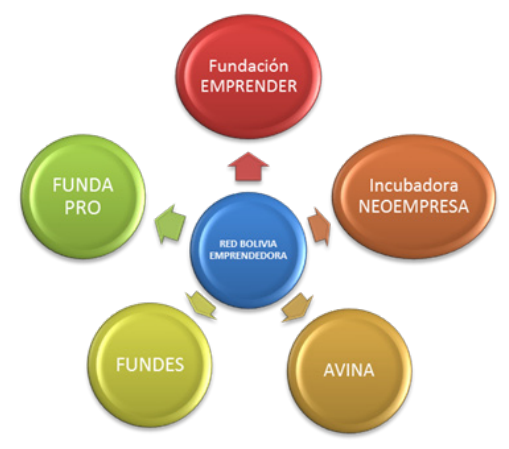

Fuente Elaboración según MEMORIA RBD 2006

\section{Gráfico 6.- Agentes que constituyeron la Red Bolivia Emprendedora}

Desde el 2009, la Red Bolivia Emprendedora se descentralizó en diez nodos departamentales coordinados por una Secretaria Técnica. Según la información de la red:

"Esta nueva estructura responde a una decisión estrategia de la RBE para lograr un mayor alcance regional, una participación más activa de los nodos y a través de estos de las instituciones que apoyan al emprendedor en Bolivia, tratando de ser más eficiente para que la red tenga mejor alcance y mayor institucionalidad."

Sin embargo, la ciudad de La Paz, ha bajado su participación, siendo más pasiva y menos eficiente, porque los participantes comenzaron a trabajar de manera aislada generando duplicidad de esfuerzos en las etapas de sensibilización y formación, cada uno con un método y un enfoque centralizado en sus intereses institucionales.
Tabla 1.- Las Políticas Gubernamentales

\begin{tabular}{|l|l|}
\hline \multicolumn{1}{|c|}{$\begin{array}{c}\text { CONTEXTO PARA } \\
\text { EMPRENDER }\end{array}$} & \multicolumn{1}{c|}{\begin{tabular}{c}
\multicolumn{1}{c|}{ INSTITUCIONES Y Y } \\
Políticas públicas
\end{tabular}} \\
\hline $\begin{array}{l}\text { No definidas de manera } \\
\text { explícita. }\end{array}$ \\
\hline $\begin{array}{l}\text { VMCyT -,Red de Incubado- } \\
\text { ras de Base Tecnológica }\end{array}$ \\
\hline $\begin{array}{l}\text { GAMLP - Programa de } \\
\text { Empresas Dinámicas, Incu- } \\
\text { badora de Empresas }\end{array}$ \\
\hline $\begin{array}{l}\text { Educación y capacitación } \\
\text { Servicios comerciales y } \\
\text { profesionales }\end{array}$ & $\begin{array}{l}\text { FUNDAPRO - Fondo para } \\
\text { la innovación }\end{array}$ \\
\hline $\begin{array}{l}\text { Apertura de mercado } \\
\text { interno }\end{array}$ & Capital humanon - Cátedras \\
\hline $\begin{array}{l}\text { Acceso a la infraestructura } \\
\text { física }\end{array}$ & No existen mecanismos \\
\hline $\begin{array}{l}\text { Innovación y transferencia } \\
\text { de Investigación y Desa- } \\
\text { rrollo }\end{array}$ & $\begin{array}{l}\text { No existen programas } \\
\text { especícos que lleve los } \\
\text { resultados de investigación } \\
\text { a la empresa }\end{array}$ \\
\hline Normas sociales y cultu- & $\begin{array}{l}\text { Concursos de ideas de } \\
\text { negocio }\end{array}$ \\
\hline
\end{tabular}

FUENTE: En base a MODELO GEM

Como se observa en la tabla, las políticas gubernamentales que apoyan a emprendimiento, no lo hacen de forma explícita. Las iniciativas se orientan hacia el apoyo a micro y pequeñas empresas establecidas, a quienes se dirigen los instrumentos deapoyo (capacitación, asistencia técnica, asociatividad, ferias y contactos con créditos blandos).

Los programas públicos que existen aun no promueven la creación de nuevas empresas y menos aun de empresas dinamicas. Se han formulado algunas líneas de acción, como el apoyo a la red Bolivia Incuba a través de la red de investigación de incubadoras de base tecnológica, que esta trabajando en aglutinar y unir a todos los interesados en la incuabcion y aceleración empresarial en el país, se han hecho ocntactos con universidades de otros países para transferencia de conocimiento tecnología y experiencia, de estas la qque mas frutos ha generado es la relación con el Instituto Politecnico Nacional de Mexico, a través de la transferencia de su modelo Robusto de Incubacion a las universidades y otras instituciones que cuenten con incubadoras de empresas o proyectos de implementacion, pero estos esfuerzos no han contado con financiamiento para implementar fondos de inversión u otras tecnologías de apoyo 
operativo a los emprendedores, su influencia se ha reducido a movilizar y sensibilizar en el tema a agentes interesados en implementar incubadoras de empresas y aceleradoras.

El Gobierno Autonomo Municipal de La PazGAMLP, esta en el proceso de implementar un programa de Empresas DInamicas, que busca acelerar a las empresas dinámicas, además cuenta con una incubadora de empresas y pretende en termino de cultura y sensibilización generar una marca ciudad emprendedora. Pero no ha generado otro tipo de apoyos para los emprendedores en el campo impositivo u otro tipo de políticas publicas.

En cuanto a los esfuerzos que se han realizado en el país en materia de formación emprendedora, se han identificado iniciativas lideradas por Universidades, $\mathrm{MpD}$, fue el pionero y el que mas experiencia y conocimiento ha acumulado en la temática del emprendimiento, través de cursos impartidos por expertos internacionales, cátedras de emprendimiento en sus programas de pre y postgrado, y transferencia de tecnología a otras instituciones, La UNSLP, ha implementado una pre incubadora y ha trabajado en pre incubación y apoyo a la elaboración de planes de negocio.

La UPEA esta en proceso de formalizar un proyecto de implementación de incubadora de empresas; la UMSA tiene una resolución rectoral de incubadora de empresas, pero aun no ha conseguido resultados; las otras universidades EMI, UDABOL, UReal, UTB, han puesto el foco en promover la cultura emprendedora mediante cátedras de emprendimiento y formación de capacidades en la elaboración de modelos y planes de negocio. Ninguna cuenta con programas de financiamiento propias.

El desarrollo de la cadena de financiamiento emprendedor es todavía débil. La principal oferta de capital semilla en la actualidad es el Fondo para la Innovacion dependiente de FUNDAPRO, (en el pasado existio el Fondo Emprender), que ofrece tecnología financiera para emprendimientos, bajo condiciones favorables, (asociación accidental, crédito convertible en acciones) y apoyo en la gestion estratégica y operacional del emprendimiento, necesita q los eslabones previos se articulen y alineen en sus necesidades y requerimientos en cuanto a la prueba de prototipos en el mercado e inicio de operaciones comerciales. Los esfuerzos de formar redes de inversión ángel todavía no dan frutos, FUNDAPRO hizo una iniciativa la Red Genesis, que no continuo operaciones.
Las diversas instituciones de micro finanzas que operan en el país, no disponen de instrumentos que sean atractivos para emprendimientos con alto potencial de crecimiento, ya que por lo general se trata de créditos por bajos montos y a elevadas tasas de interés. La banca comercial tampoco ha generado instrumentos dirigidos a los emprendedores en etapas iniciales, a quienes considera de riesgo alto.

La falta de apoyo al desarrollo de iniciativas empresariales de alto valor se ve reflejado en la existencia de contados emprendedores llamados "exitosos", y una característica común de ellos, es que en su camino al éxito, no contaron con el soporte de instituciones y organizaciones de apoyo emprendedor. Declaran que "lo lograron por sus propios medios.

Las incubadoras de empresas responden a esfuerzos aislados deorganizaciones no gubernamentales y de universidades, las que no cuentan actualmentecon financiamiento público para sus operaciones que garantice su sostenibilidad.

La incubadora del GAMLP, provee espacio físico y seguimiento al emprendedor sin costo por permanencia a la fecha cuenta con dos empresas en proceso de egreso como éxito de sus operaciones, la incubadora Ifaro, cobra una tasa mensual al emprendedor en el campo del cotrabajo, y provee también asesoría en conocimiento gerencial para la toma de decisiones, a la fecha ha egresado 7 empresas desarrolladoras de software y tiene dos en proceso de incubación, esta trabajando en buscar construir un fondo de capital angel, ya que esta ha sido su mayor deficiencia.

Entemas detransmisión de cultura emprendedorase considera importante las competencias nacionales de planes de negocio, "ideas emprendedoras", la que se desarrolla en forma anual desde el 2004, a pesar de ser un cocncurso dirigido a todos los segmentos del emprendimiento, usualmente unas tres categorías de premios estaban enfocados en emprendimientos basados en tecnologia. "Innova Bolivia", es el otro concurso nacional comandado por la Fundacion Maya, enfocado exclusivamente al mercado de emprendimientos de tecnología e innovacion y tiene alcance nacional.

Así mismo, existe el aporte a nivel de universitarios con los eventos de Startup.bo y HUB7, que organizan el formato de creación de empresas conocido como StartupWeekend, cuyos ganadores pueden acceder a competencias mundiales para acceder a financiamientos y también alimentan a 
las incubadoras con nuevas ideas de negocio, las versiones 2013 contaran con el apoyo de la Red Bolvia Emprendedora, y en el caso de la ciudad de La Paz, apoyo de la GAMLP.

Es recalcable meniconar que los agentes del ecosistema también están conectados con programas de emprendimiento y participan en los mas importants foros de emprendimiento a nivel internacional.

Estas reflexiones se resumen en el siguiente cuadro:

Tabla 2.- Elementos y Factores que inciden sobre el proceso emprendedor

\begin{tabular}{|l|c|}
\hline $\begin{array}{c}\text { Elementos y Factores } \\
\text { que inciden sobre el } \\
\text { proceso emprende- } \\
\text { dor }\end{array}$ & INCIDENCIA \\
\hline $\begin{array}{l}\text { Las condiciones socia- } \\
\text { les y económicas }\end{array}$ & + \\
\hline $\begin{array}{l}\text { Cultura y sistema } \\
\text { educativo }\end{array}$ & ++ \\
\hline $\begin{array}{l}\text { Estructura y dinámica } \\
\text { productiva }\end{array}$ & +++ \\
\hline Aspectos personales & - \\
\hline $\begin{array}{l}\text { Redes del emprende- } \\
\text { dor }\end{array}$ & +++ \\
\hline Mercado de factores & -- \\
\hline $\begin{array}{l}\text { Regulaciones y polí- } \\
\text { ticas }\end{array}$ & -- \\
\hline
\end{tabular}

Fuente: Elaboración Propia en base a KANTIS, 2004.

\section{3.- PROPUESTA DE MAPEO INSTITUCIONAL}

Una vez identificados los agentes, sus actividades y necesidades, se ha considerado para la presente propuesta las siguientes etapas clave: la sensibilización, de cultura emprendedora de empresas dinámicas; la pre-incubación, que dota de emprendedores sensibilizados a las incubadoras de empresas; las incubadoras que prueban a las start ups en la modelación - prototipación del negocio; las aceleración de nuevas empresas dinámicas que necesitan abrir nuevos mercados y los fondos de inversión que acompañan el escalamiento de la start up.
Estas etapas serán alineadas por los siguientes ejes institucionales trasversales a cada etapa, donde se reconocen los esfuerzos que deberían impulsar y apoyar a nuevas empresas dinámicas, facilitándoles modelos de transferencia, apoyo en habilidades gerenciales y acompañamiento de instituciones con experiencia.

Para complementar este mapa se recomienda también la adaptación de las instituciones de financiamiento, con el fin de elevar el acceso a préstamos de capital financiero adecuado a las características del emprendedor, así como también mejorando la legislación para posibilitar el acceso a mercados $y$ facilidades de incorporación $y$ formalización de las empresas, exenciones tributarias temporales, que posibiliten mejores condiciones de crecimiento en las etapa inicial de las start ups.

\section{4.- Mapeo de agentes $y$ redes para la consolidación de un Ecosistema}

Para crear el mapa se ha considerado la información obtenida en la investigación de campo, la cual ha sido sistematizada en los ejes temáticos:

- $\quad$ En el eje vertical el Modelo de ABIIGS (ver pág del presente documento), por adaptarse mejor a la necesidad de identificar y organizar a las instituciones en sus áreas de interés y de incidencia, que es el interés del presente trabajo de investigación, para facilitar la lectura del mapa. Donde se considera a las Instituciones Académicas, Banca e Instituciones Financieras, Infraestructura e industrias relacionadas, Políticas Gubernamentales, Capital social, cultural, drivers políticos, con los que se realiza un diagnóstico de la situación actual en cuanto al fomento de las empresas dinámicas.

- El eje vertical etapas del proceso emprendedor expuestas anteriormente (ver pag. ) Considerando que el proceso emprendedor es multidimensional, estas etapas son genéricas a diferentes visiones del tema dentro de los agentes del ecosistema. Así mismo se consolidó los subprocesos que tienen una lógica en el tiempo y el espacio del emprendedor. 


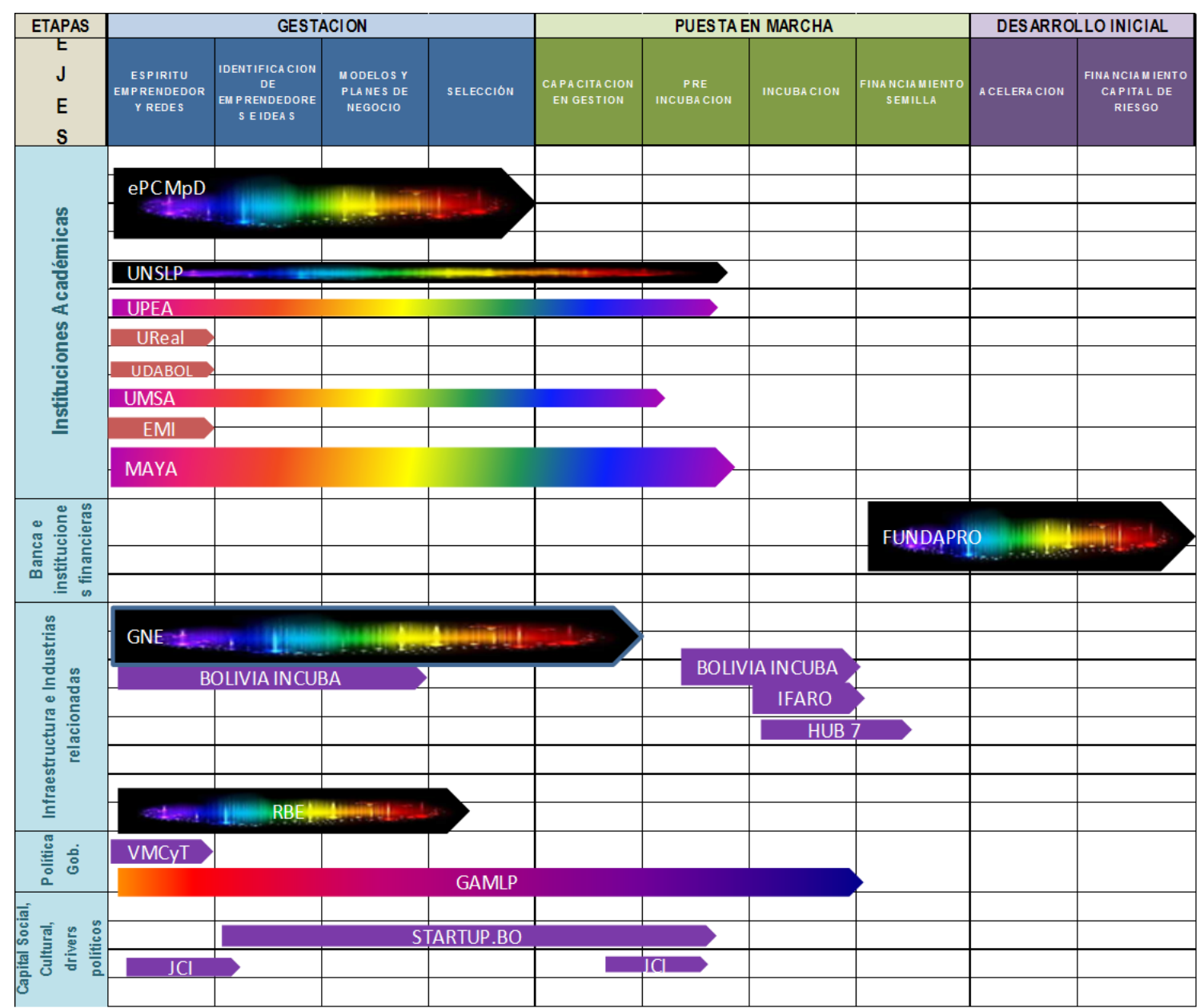

Fuente: Elaboración propia

Gráfico 7.- Mapeo de agentes y Redes para la consolidación de un Ecosistema que apoye a las Empresas Dinámicas

El mapa debe ser leído desde el cuadro de percepción (ver anexo), donde el color refleja el número de años, experiencia y el ancho como es percibida cada institución por sus pares en el ecosistema, el color negro a las que mejor son percibidas en experiencia; el degrade de colores a las q tienen poco más de 5 años de experiencia y están acumulando experiencia; las de color puro tienen menos de 5 años de experiencia y son las mas noveles en el ecosistema.

La academia solo esta trabajando en cátedras de emprendimiento, debería como en el caso de ePC MpD generar experiencias en identificación, modelado y selección de emprendedores, en primera instancia en trabajo coordinado con las preincubadoras e incubadoras como en el caso de UNSLP, MAYA, UMSA, UPEA esta iniciando sus primeros pasos en procesos de preincubacion e incubación.

Solo existe el fondo de la innovación para proveer de financiamiento a las empresas en etapas iniciales, HUB7 está gestionando un fondo pero a pesar de tener incidencia en el ecosistema de la ciudad de La Paz, este operaria físicamente en Cochabamba, capitales ángeles son inexistentes.

Solo operan dos incubadoras en la ciudad de La Paz; IFaro y la incubadora del GAMLP, las universidades deberían iniciar procesos de implementación de mas incubadoras, la Red Bolivia Incuba provee de transferencia tecnológica a incubadoras en funcionamiento o proyectos de implementación interesadas.

El VMCyT, provee el soporte institucional desde el gobierno estatal para las iniciativas, programas $\mathrm{y}$ proyectos que tengan las incubadoras y se constituye en un nodo que posibilita el contacto con otros gobiernos y agencias de cooperación, actualmente se trabaja con los modelos de transferencia en incubación del IPN Mexico; el GAMLP, a través de sus programas de cultura emprendedora y empresas dinámicas busca lanzar a La Paz como una ciudad emprendedora. 
Startup.bo es la única iniciativa desde el voluntariado que trabaja en modelar ideas de negocio para luego enviarlas a procesos emprendedores, realiza el startup weekend en promedio dos veces al año y en convenio con incubadoras genera oportunidades para nuevos emprendedores, la JCI, fomenta entre sus miembros la generación de ideas y busca tener procesos de preincubacion.

La figura muestra los vectores de cada institución, donde se ha considerado:

1. El origen desde el eje vertical clasificado en ABIIGS.

2. La dirección de cada institución según su orientación en el trabajo por etapas.

3. El módulo o tamaño del vector, por tiempo de experiencia.

4. El sentido representa hacia dónde va dirigido sus resultados, esto permite interpretar donde comenzaría la vinculación

\section{7.- RECOMENDACIONES}

La realización de talleres de co-construcción y desarrollo de visión conjunta ha facilitado la emergencia de una fértil instancia de conocimiento mutuo y generación de complicidad para colaborar entre actores diversos. También mediante el desarrollo de planes de acción concretos que mejoren medidas de Doing Business y la generación de iniciativas gubernamentales o público-privadas que mejoren el clima de negocios para emprender, en general o en ámbitos específicos que se consideren como prioritarios en cada caso. Bajo este enfoque, se ha realizado un trabajo fuerte en los países de la región, desarrollando como punto de partida, un mapa de ecosistema de emprendimiento y planes de acción para el desarrollo de este ecosistema, incluyendo actores clave, interacciones y alianzas necesarias para lograr nutrir a emprendedores del valor que necesitan para desarrollar sus empresas.

Bajo esta experiencia empírica, se ha identificado algunas consideraciones al momento de definir el ecosistema de apoyo emprendedor para un país:

- Generar equipos de trabajo heterogéneos, de tal manera que el ejercicio de definición del Ecosistema posea multiplicidad de miradas $\mathrm{y}$ enfoques, lo que permita enriquecer $\mathrm{y}$ complementar la mirada que podría dar una sola institución. Esto implica hacer partícipes de estas sesiones a instituciones tanto públicas como privadas y dentro de estas instituciones, a personas con distinto grado de conocimiento e involucramiento con la actividad emprendedora y empresarial.

- Establecer un lenguaje común, al inicio de la sesión, de tal manera de evitar las ambigüedades y orientar el trabajo hacia un objetivo definido para todo el grupo de trabajo.

- Generar consenso, en cuáles son los ámbitos del ecosistema más relevantes para la realidad del país o ciudad para el cual se está desarrollando la actividad. La búsqueda de consenso permite involucrar a todos los actores en torno a definiciones que son comunes y aceptadas por todos los actores. Este punto resulta fundamental, pues para lograr el consenso se requiere un espacio de conversación y confianza que lo constituye una red de trabajo en un enfoque de cocreación, considerando que son actores de la mayor relevancia en los negocios o sector público que no siempre tienen la oportunidad de conversar y alinear una mirada de futuro con otros actores.

- Definir mosaico o mapa de aliados, identificando instituciones relevantes para cada ámbito del ecosistema y manteniendo un vínculo permanente con ellos a través de sesiones de trabajo y flujo de información que los mantiene actualizados, amén de conversaciones y visitas uno a uno para ir mejorando la disposición e involucramiento en la acción.

- Definir espacios de colaboración en la lógica de ganar-ganar con los aliados. En la lógica de orquestación de valor, generar interacciones ganar-ganar con instituciones de servicios complementarios no es un trabajo fácil, pero existen experiencias donde se han logrado alianzas de este estilo89, entendiendo que una institución que apoya emprendimiento de alto potencial, es poseedor de "futuros clientes" para instituciones de servicios empresariales. Esto refuerza el hecho de que el foco en emprendimiento de alto impacto es relevante a la hora de generar ofertas para los aliados y miembros del ecosistema.

- Diseño de planes de acción y ejecución de los mismos, para poner en actividad al ecosistema, definiendo plazos, responsables y recursos involucrados. 


\section{8.- BIBLIOGRAFÍA}

Fernandez, Marco Antonio, (2010). GLOBAL ENTREPRENEURSHIP MONITOR Reporte Nacional Bolivia,

KantisAngelelli,(2005), DESARROLLO EMPRENDEDOR, América Latina y la experiencia internacional,

Neck, H. M., Meyer, G. D., Cohen, B., \& Corbett, A. C. (2004).An entrepreneurial system view of new venture creation. Journal of Small Business Management,

Park, J. S. (2005). Opportunity recognition and product innovation in entrepreneurial hi-tech start-ups: a new perspective and supporting case study. Technovation

\section{9.- REFERENCIAS}

[1] MEMORIA VMCYT: REDES NACIONALES DE INVESTIGACIÓN CIENTÍFICA Y TECNOLÓGICA, Red Nacional de Incubadoras de Empresas de Base Tecnológica, pág. 95, (2011).

[2] FERNANDEZ, MARCO ANTONIO GEM Bolivia Encuesta a la población Adulta (APS) 2010, pág 25.

[3] El proyecto de investigación The Global Entrepreneurship Monitor, GEM, se inició con el estudio de 10 países, hasta llegar a 59 en el 2010, con 175.000 personas encuestadas. Este grupo de países cubre más del $52 \%$ de la población mundial y el $84 \%$ de la producción mundial.

[4] ISENBERG, Daniel, 2012.

[5] ISENBERG DANIEL, The Big Idea: How to Start an Entrepreneurial Revolution, pág. 14. (2010).

[6] Business Model Canvas El lienzo de modelo de negocio es una herramienta de generación de modelos de negocio ideada por Alexander Osterwalder e Yves Pigneur que, basado en el concepto «lienzo estratégico» de W. Chan Kim y Renée Mauborgne, permite describir de manera lógica la forma en que un modelo de negocio en marcha o recién iniciado crea, entrega y captura valor.

[7] Expulsión de Organizaciones internacionales como USAID.
[8] Trabajó en la Incubadora de empresas NEOEMPRESA, actualmente coordina el Fondotec. 